FORBES \& FIFTH

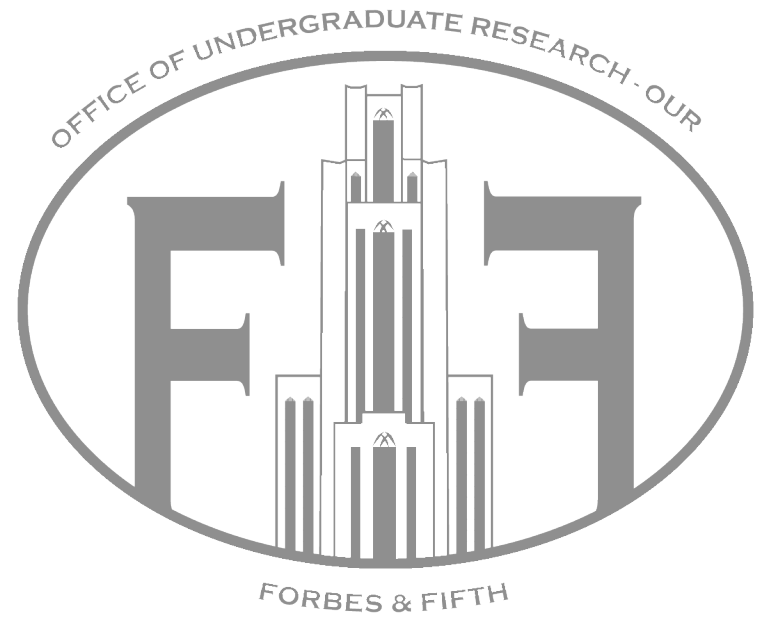




\section{Schizophrenia and a Means to Study It}
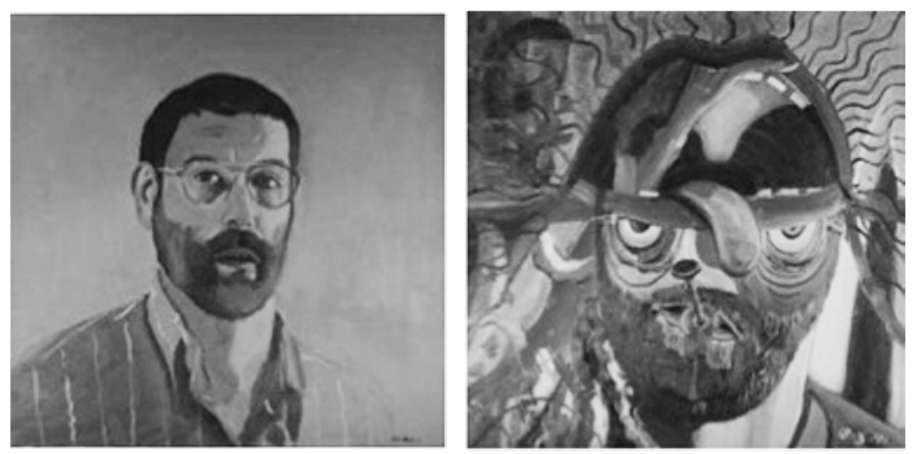

The two paintings depicted above were created by Bryan Charnley, an aspiring painter who suffered two mental breakdowns during his time at art school. Bryan was later diagnosed with schizophrenia. Starting in 1991, he began to paint portraits to express his feelings during his treatments. ${ }^{1}$ The painting on the left was made in early April, 1991. During this time, he was heavily medicated with antipsychotics. The painting on the right was made a month later, after his medication dosage was reduced. He wrote about his noticeable shift in expression: "I

"'I feel I am always divided against myself by myself. Again, the nail in the mouth expresses my social ineptitude and an inability to socialize which makes me a target." feel I am always divided against myself by myself. Again, the nail in the mouth expresses my social ineptitude and an inability to socialize which makes me a target. Still I feel I am giving off strong personality vibrations, hence the wavy lines emanating from my head."'2 Bryan committed suicide in July, 1991. It is clear from Bryan's written accounts and his artwork that schizophrenia altered his perceptions and distorted reality.

Schizophrenia is a neurodevelopmental disorder that affects $0.5 \%-1.0 \%$ of the world's population. ${ }^{3}$ The disease usually comes to clinical attention around late adolescence and early adulthood. Around 
$10 \%$ of patients will eventually commit suicide, while most will eventually experience some form of lifetime disability. To be diagnosed with schizophrenia, a patient must meet certain criteria established by the American Psychiatric Association, which are elucidated in the Diagnostic and Statistical Manual of Mental Disorders (DSM). ${ }^{4}$ Symptoms of schizophrenia are generally grouped into three domains: 1) Positive symptoms - disordered thoughts and speech, sensory hallucinations (perceptual disturbances), and delusions (false beliefs that are held despite contradictory evidence); 2) Negative symptoms - flat emotion, alogia (lack of speech), anhedonia (lack of pleasure from normal activity), and asociality (lack of motivation to engage in social behavior); 3 ) Cognitive symptoms - deficits in cognitive control, which is the ability to plan, initiate and control goal directed behavior. ${ }^{3}$ Once diagnosed, antipsychotic medications are usually given as the first course of treatment. Currently, there are medications available which alleviate the positive and negative symptoms, but there is no effective treatment for cognitive dysfunction. So while positive and negative symptoms are most commonly associated with the illness and are more or less treatable, recent evidence suggests that the deficiencies in cognitive domains are the core dysfunction of the illness. ${ }^{5}$

Cognitive control, as the name implies, is regulated by the brain. The human brain is divided into many different areas made up of specific circuits that are responsible for integrating and processing different streams of information; these circuits are comprised of neurons, which are the basic fundamental units of the nervous system. Neurons communicate with each other using signaling molecules, and can be divided into two basic classes: excitatory and inhibitory. The major way this communication occurs is through the release of signaling molecules that bind to other neurons in proximity. The majority of excitatory neurons use glutamate as their signaling molecule, while the majority of inhibitory neurons use $\gamma$-aminobutyric acid (GABA). A unique aspect of neurons is that they have a specific voltage threshold that, when crossed, causes them to have a flood of activity that results in communication with other neighboring cells. Excitatory neurotransmitter input causes a neuron to approach its voltage threshold, and inhibitory input causes it to recede from the threshold. Whether a neuron crosses its threshold, causing the release of its signaling molecules, depends on the amount of 
excitatory and inhibitory inputs onto it.

The communication between these neurons within specific circuits allows for specific tasks to be accomplished. The coordinated activity of neurons within these circuits is imperative for processing information, because without it, the system would go haywire. Think of the brain as one giant machine with many individual parts that perform specific functions to communicate signals. Connections between the parts form a specific local circuit, which in turn is connected to another circuit, and the integrity of the connections between the circuits is what determines the processing power of the machine. Thus, if the functional integrity of the machine is compromised, the system goes awry. This is the basic idea behind cognitive dysfunction in schizophrenia. Therefore, by studying how neurons communicate within the circuit we can try to understand what "parts of the machine" are altered in schizophrenia. In the brain, cognitive control, in part, is achieved through the coordinated excitatory and inhibitory activity of neurons within the dorsolateral prefrontal $\operatorname{cortex}^{6}$ (DLPFC):

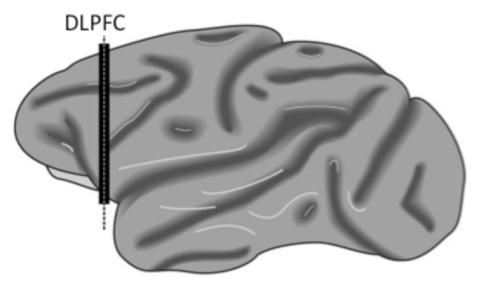

Within the machinery of the DLPFC, the fundamental unit is the neuron:

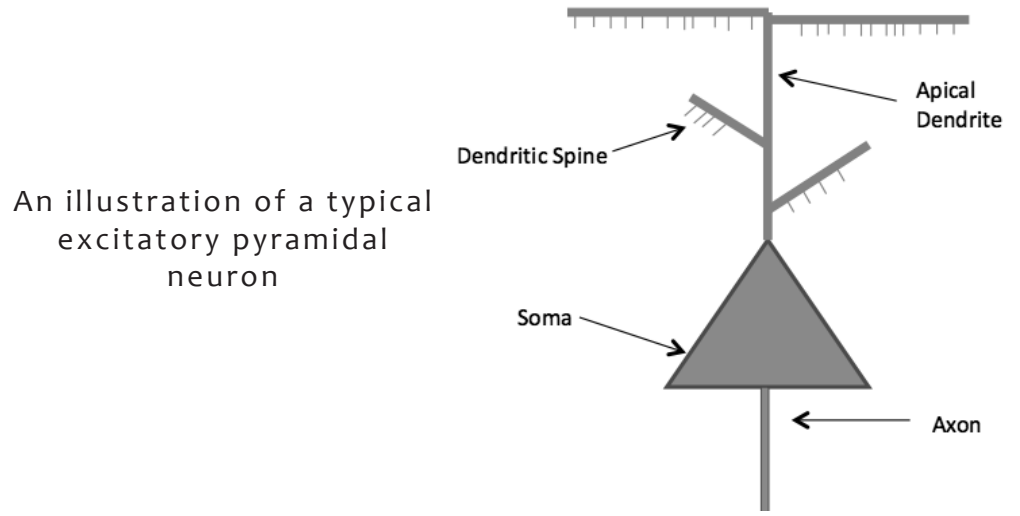


The soma of a neuron is the cell body, where the majority of the cell's organelles reside. Extending off of the soma are two specialized kinds of projections: the axon and the dendrite. Dendrites are branched projections that serve to accumulate and conduct the electrochemical input received from other neurons to the soma. Dendrites may have spines, which are protrusions that typically receive inputs from other neurons. The other projection, the axon, is where the flood of activity occurs once a neuron reaches threshold. This activity races down the axon until it reaches the axon terminal, where it stimulates the release of neurotransmitters onto other neurons.

Excitatory pyramidal neurons receive excitatory and inhibitory inputs from neighboring pyramidal neurons and inhibitory interneurons, respectively. An overwhelming majority of excitatory inputs occur on the dendritic spines of pyramidal neurons, whereas inhibitory inputs are located across the entire neuron. Excitatory effects onto pyramidal cells occur through glutamate signaling and inhibitory effects onto pyramidal cells occur through $\gamma$-aminobutyric acid (GABA) signaling. This simply means that an excitatory cell releases glutamate onto pyramidal cells to excite them, and an inhibitory cell releases GABA onto the pyramidal cells to inhibit them. The overall output of the neuron, then, depends on the integration of excitatory and inhibitory inputs within a given time frame.

While there are many ways to study the brain, a technique called immunofluorescence microscopy is especially useful for visualizing the different parts that make up neuronal circuits. This technique requires a brain, and the brains used are ones that have been graciously donated by families of deceased individuals. Once a human brain is obtained, various brain areas (like the DLPFC) are sliced and stored in a special preservative solution. Then, once slices have been obtained, the next step of the process is to run immunohistochemistry (or staining) on the slices of brain tissue. Basically, this allows for the labeling of different components of the neuronal circuits with different colors. By having different components labeled with different colors, the visualization and differentiation between components becomes possible. The tissue is then placed on a fluorescent microscope and images of the stained tissue are taken. The process of image acquisition is long and tedious, but these images provide a snapshot of intact neuronal circuits that can be 
subjected to three-dimensional analysis.

Three-dimensional analysis can provide important information about the circuit in question: the components of the circuit can be highlighted, the number of components within a circuit can be measured, the communication targets of certain components can be determined, the actual length and width of components can be measured, and the relative levels of components within other components can be measured. This type of analysis is extremely powerful when trying to examine disease states like schizophrenia. With immunofluorescence microscopy, a normal circuit can

"Does a person with schizophrenia have fewer parts? Is there the same number of parts but altered expression within those parts? If, in a normal circuit, a component consists of other parts, are all the other parts altered or only a handful?" be examined and assessed. Then, diseased brain tissue can be analyzed in the same way and be compared to the normal tissue. This allows for the discovery of alterations within these circuits. Does a person with schizophrenia have fewer parts? Is there the same number of parts but altered expression

within those parts? If, in a normal circuit, a component consists of other parts, are all the other parts altered or only a handful? These are the sorts of questions that are asked in my work lab.

Immunofluorescence microscopy and other experimental techniques have shown that both markers of excitation and inhibition are altered in the DLPFC in patients with schizophrenia. For example, in schizophrenia, there is a twenty percent decrease in the density of dendritic spines in the DLPFC, which suggests altered excitatory signaling. ${ }^{89}$ Furthermore, markers of inhibitory signaling are altered in schizophrenia as a result of altered gene products and proteins. ${ }^{10,11,12}$

Research is ongoing and the specific aspects of these systems are being examined. The ultimate goal is to characterize every unique circuit in the DLPFC. By characterizing unique circuits by the number of parts, their specific targets, and their development, diseases like schizophrenia can be pinpointed to alterations in aspects of the overall circuit. Alterations in the circuit can mean there are communication problems 


\section{FORBES \& FIFTH}

and thus synchrony problems. Once these specific changes are identified, they can then be used to develop specific medications that attempt to alleviate the symptoms "I realised that the fear caused by the anger...is causing me to hallucinate telepathy... Can this ever change or will the anger always remain?"” associated with certain neurological diseases. Bryan Charnley wrote the following on June $27^{\text {th }}$, 1991: "I realised that the fear caused by the anger...is causing me to hallucinate telepathy...Can this ever change or will the anger always remain?"7 My hope is that through the research I conduct in my lab, I can contribute to an ever-growing body of knowledge that will eventually aid in helping to restore the brain back to its optimum function, so that people like Bryan Charnley can better cope with their psychological illnesses. 
SHEIKH 